\title{
Apatinib as first-line treatment in patients with advanced hepatocellular carcinoma: a phase II clinical trial
}

\author{
Zhenyu Hou, Keyun Zhu, Xuejiao Yang, Ping Chen, Wei Zhang, Yunlong Cui, Xiaolin Zhu, \\ Tianqiang Song, Qiang Li, Huikai Li, Ti Zhang
}

Department of Hepatobiliary Surgery, Tianjin Medical University Cancer Institute and Hospital, Key Laboratory of Cancer Prevention and Therapy, National Clinical Research Center for Cancer, Tianjin Clinical Research Center for Cancer, Tianjin, China

Contributions: (I) Conception and design: T Zhang, H Li; (II) Administrative support: Q Li, T Song; (III) Provision of study materials or patients: T Zhang, H Li; (IV) Collection and assembly of data: Z Hou, K Zhu, X Yang, P Chen, W Zhang; (V) Data analysis and interpretation: Z Hou, X Zhu, Y Cui; (VI) Manuscript writing: All authors; (VII) Final approval of manuscript: All authors.

Correspondence to: Ti Zhang, MD, PhD; Huikai Li, MD. 24 Bin Shui Road, Hexi District, Tianjin, China.

Email: zhangti@tjmuch.com; tjchlhk@126.com.

Background: The prognosis for advanced hepatocellular carcinoma (HCC) remains clinically unsatisfying. Apatinib has proven to be a very effective treatment for advanced HCC in our previous retrospective study. Our aim in this study was to evaluate the efficacy, safety, and toxicity of apatinib in patients with advanced HCC.

Methods: This single-arm, open-label phase II clinical trial enrolled patients with advanced HCC. These patients received apatinib, $500 \mathrm{mg}$ once daily, until disease progression, unacceptable toxicity, consent withdrawal, or death. One treatment cycle consisted of 4 weeks of apatinib treatment. The response evaluation criteria in solid tumors (RECIST) was used to assess tumor response every 1-2 cycles. The primary endpoint was the objective response rate (ORR), while the secondary endpoints were the overall survival (OS), progression-free survival (PFS), disease control rate (DCR), and toxicity.

Results: Between December 2016 and June 2018, 23 patients were enrolled in the study, 22 of whom were available for response evaluation. The cutoff date was August 10, 2018. The overall ORR and DCR were 30.4\% and 65.2\%, respectively. The median OS and PFS were 13.8 (95\% CI: 5.3-22.3) and 8.7 (95\% CI: 5.9-11.1) months, respectively. The most common treatment-related adverse events were proteinuria (39.1\%), hypertension (34.8\%), and hand-foot-skin reaction (34.8\%).

Conclusions: Apatinib showed robust clinical activity in patients with advanced HCC. Moreover, apatinib was safe to use, well tolerated, and had acceptable toxicity. (NCT03046979).

Keywords: Apatinib; hepatocellular carcinoma (HCC); angiogenesis; vascular endothelial growth factor (VEGF); tyrosine kinase

Submitted Mar 30, 2020. Accepted for publication Jul 30, 2020.

doi: 10.21037/atm-20-2990

View this article at: http://dx.doi.org/10.21037/atm-20-2990

\section{Introduction}

Hepatocellular carcinoma (HCC) is the fifth most common type of cancer and the third highest cause of cancer-related mortality worldwide (1). The prognosis of HCC especially in its advanced stages, is poor overall. Systemic therapy using cytotoxic agents has not provided a survival benefit (2), and the recommended first-line treatment for advanced HCC is sorafenib and lenvatinib (3). However, in the SHARP and ORIENTAL clinical trials, the partial response (PR) rate for sorafenib was only $2 \%$ and $3.3 \%$, respectively, with an overall survival (OS) of only 10.7 and 6.8 months, respectively $(4,5)$. Meanwhile, the REFLECT study reported that lenvatinib yielded a slightly higher median OS 
of 13.6 months and showed similar efficacy as sorafenib (6).

Angiogenesis plays an important role in tumorigenesis and development (7). Vascular endothelial growth factor (VEGF) signaling is considered to be the main pathway responsible for angiogenesis (8-11), with VEGFR2 being a critical mediator of VEGF-induced angiogenic signaling (12). Apatinib is a novel oral angiogenesis inhibitor that targets the intracellular ATP binding site of VEGFR2. As a VEGFR2-specific inhibitor, apatinib has been approved for use in gastric cancer as a third-line treatment (13). Recent studies have also shown that several other types of cancers are responsive to apatinib treatment, including ovarian, breast, and non-squamous non-small cell lung cancer (14-16). The efficacy of apatinib in treating advanced liver cancer has drawn wide interest. In our previous retrospective study, apatinib proved to be highly effective for the treatment of advanced HCC, achieving a PR rate of $40.9 \%$ (17). However, selective bias could not be avoided as this was a retrospective study. Therefore, a phase II clinical trial was conducted to further confirm the efficacy and safety of apatinib as first-line treatment in patients with advanced HCC. We present the following article in accordance with the TREND reporting checklist (available at http://dx.doi.org/10.21037/atm-20-2990).

\section{Methods}

All HCC patients eligible for this phase II clinical trial were informed of the trial details and provided written informed consent before participating. The study was conducted in accordance with the Declaration of Helsinki (as revised in 2013). The study was approved by the Ethics Committee of the Tianjin Medical University Cancer Institute and Hospital and is registered on clinicaltrials.gov (NCT03046979).

Patient inclusion criteria included age $\geq 18$ years; presence of portal vein tumor thrombosis (PVTT) or distant metastasis; Child-Pugh class A; presence of at least one measurable lesion as defined by the Response Evaluation Criteria in Solid Tumors (RECIST1.1); an Eastern Cooperative Oncology Group (ECOG) performance status of $0-2$; adequate hematologic, coagulation, hepatic, renal, and cardiac function; and willingness to practice contraception during the study period. The exclusion criteria included concurrent malignancies; Child-Pugh score $\geq 7$ or severe uncontrollable ascites; severe cardiovascular disease; uncontrolled high blood pressure; history of human immunodeficiency virus infection; clinically serious infection [grade $>2$, according to the Common Terminology Criteria for Adverse Events (CTCAE), version 4.0]; seizures requiring medication (steroid or antiepileptic drugs); history of allogeneic organ transplantation; signs or history of bleeding disorders; current renal dialysis treatment; metastatic liver cancer; uncontrolled ascites (that are not easily controlled by diuretic drugs); encephalopathy; history of gastrointestinal bleeding within 30 days prior to enrollment; and a history of bleeding esophageal varices without receiving effective therapy or treatment.

\section{Study design and treatment}

This was an open-label, single-arm, investigator-initiated phase II clinical trial, conducted in Tianjin Medical University Cancer Institute and Hospital. The trial oversight, database management, and quality assurance were performed at the Department of Hepatobiliary Surgery at Tianjin Medical University Cancer Institute and Hospital. Apatinib was provided by Jiangsu Hengrui Medicine Co., Ltd. Patients received oral apatinib at home until disease progression, unacceptable toxicity, consent withdrawal, or death. A starting dose of apatinib $500 \mathrm{mg}$ was administered, once daily. Dose reduction was permitted to $250 \mathrm{mg}$ once daily or $250 \mathrm{mg}$ once every 2 days if patients experienced grade 3/4 adverse events (AEs), at the investigator's clinical discretion. One treatment cycle was defined as apatinib treatment for a period of 4 weeks.

\section{Outcome evaluation}

Objective response rate (ORR) was the primary endpoint in this study. The secondary endpoints were OS, progressionfree survival (PFS), disease control rate (DCR), and toxicity. PFS is defined as the time from the start of treatment to the onset of disease progression. OS is defined as the time from the start of treatment to the death. ORR is defined as the proportion of patients achieving complete or PR according to RECIST criteria.

Enhanced computed tomography (CT) and magnetic resonance imaging (MRI) were used to assess tumor response at an interval of 1-2 months. In patients with PVTT, Vp3 was defined as invasion of a tumor thrombus into the first branch of the portal vein, and $\mathrm{Vp} 4$ was defined as invasion of a tumor thrombus into the portal trunk or extending to a branch on the contralateral side (18). Based on the RECIST criteria (version 1.1), responses were defined as complete response (CR), PR, stable disease 


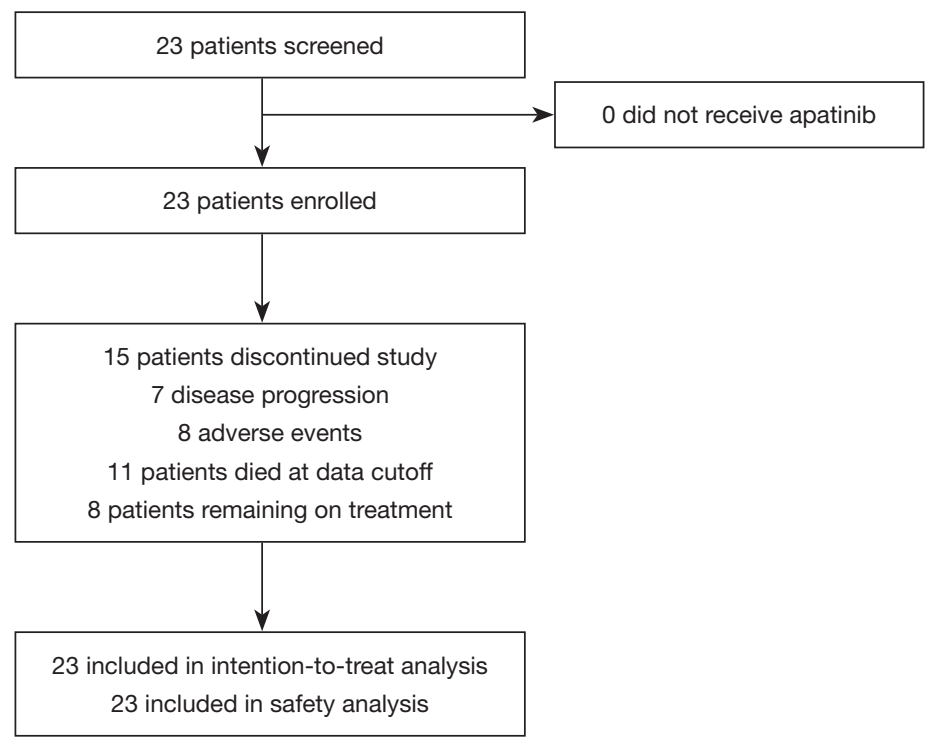

Figure 1 Trial profile.

(SD), and progressive disease (PD). AEs were classified and graded according to the CTCAE (version 4.0). Follow-up investigations were conducted monthly to determine the survival status, duration of tumor progression until death, or withdrawal of the patient from the study. The monthly follow-up procedures also comprised physical examination, assessment of ECOG performance, tumor markers, and quality of life (EORTC QLQ-C30).

\section{Statistical analysis}

Simon's optimal two-stage design was used to test the sample size. Our hypothesis was that the proportion of patients achieving an overall response would be $\leq 5 \%$, while the alternative hypothesis was that the proportion would be $\geq 30 \%(\alpha=0.05, \beta=0.20)$. Based on these hypotheses, a total sample size of 18 patients was required for evaluation of the primary endpoint. Overall, the treatment regimen would be considered statistically significant if a total of two or more responses were observed. The estimated sample size was determined to be 23 patients, which accounted for dropouts and withdrawals. Patients who received at least 1 month of apatinib were included in the survival and safety analysis, which was performed on the intention-to-treat population. The Kaplan-Meier method was used to estimate OS and PFS and associated $95 \%$ confidence intervals (CIs), while log-rank test was used to compare the OS and PFS in subgroups. Statistical analyses were performed using SPSS 22.0 (SPSS Inc., Chicago, IL, USA).

\section{Results}

Between December 2016 and June 2018, a total of 23 patients were enrolled into the study (Figure 1). The characteristics of patients forming our study group are reported in Table 1. There were 18 male patients and 5 female patients, with a median age of 54.1 years. There were 8 patients with PVTT, 5 (21.8\%) with Vp3, and 3 (13.0\%) with Vp4. For metastasis type, 17 patients (73.9\%) had distant metastasis, among whom 12 (52.2\%) had lymph node metastasis, 6 (26.1\%) had lung metastasis, and $3(13.0 \%)$ had bone metastasis. Meanwhile, 15 patients (65.2\%) had hepatitis B, and 1 (4.3\%) had hepatitis C, with the infection status being well-controlled in all patients. Finally, 17 patients $(73.9 \%)$ had abnormally high serum alpha-fetoprotein, with the AFP being $>400 \mathrm{ng} / \mathrm{mL}$ in 10 of these patients (43.5\%). The median duration of follow-up was 7.2 months.

\section{Efficacy and survival analysis}

Twenty-two patients were eligible to respond to our evaluation, with one patient withdrawing from the study before clinical evaluation. By the cutoff date of August $10,2018,11$ patients $(47.8 \%)$ had died and $15(65.2 \%)$ of the 23 patients had withdrawn from the study, with only 8 patients $(34.8 \%)$ remaining on treatment. Among the 15 patients who withdrew from the study, 7 (46.7\%) patients discontinued participation due to disease progression. The 
Table 1 Baseline characteristics of the 23 patients with advanced hepatocellular carcinoma

\begin{tabular}{|c|c|}
\hline Characteristic & $\mathrm{N}(\%)$ \\
\hline Age, years & 54.1 \\
\hline Median & $30-70$ \\
\hline \multicolumn{2}{|l|}{ Range } \\
\hline \multicolumn{2}{|l|}{ Sex } \\
\hline Male & $18(78.3)$ \\
\hline Female & $5(21.7)$ \\
\hline Child-Pugh class & $23(100.0)$ \\
\hline A & 0 \\
\hline \multicolumn{2}{|l|}{ B } \\
\hline Positive hepatitis status & $16(69.6)$ \\
\hline HBV & $15(65.2)$ \\
\hline $\mathrm{HCV}$ & $1(4.3)$ \\
\hline PVTT & $8(34.8)$ \\
\hline Vp1 & 0 \\
\hline Vp2 & 0 \\
\hline Vp3 & $5(21.8)$ \\
\hline Vp4 & $3(13.0)$ \\
\hline Distant metastasis & 17 (73.9) \\
\hline Lymph node & 12 (52.2) \\
\hline Lung & $6(26.1)$ \\
\hline Bone & $3(13.0)$ \\
\hline \multicolumn{2}{|l|}{ AFP $>$ ULN } \\
\hline Yes & $17(73.9)$ \\
\hline No & $6(26.1)$ \\
\hline \multicolumn{2}{|l|}{ ECOG-PS } \\
\hline 0 & $17(73.9)$ \\
\hline 1 & $6(26.1)$ \\
\hline
\end{tabular}

HBV, hepatitis B virus; HCV, hepatitis C virus; PVTT, portal vein tumor thrombosis; AFP, alpha-fetoprotein; ULN, upper limit of normal; ECOG-PS, Eastern Cooperative Oncology Group performance status.

remaining $8(53.3 \%)$ withdrew as a result of AEs. Overall, CR was not achieved in any patient. PR was confirmed in 7 patients (30.4\%), with SD identified in $8(34.8 \%)$ and 7 (30.4\%) meeting the criterion for PD. Tumor shrinkage was noted in 18 ( $78.3 \%)$ of 23 patients who had at least one post-baseline efficacy assessment (Figure 2A). Changes in tumor size are shown in Figure $2 B$. The overall ORR was $30.4 \%$, with a DCR of $65.2 \%$ (Table 2). The median OS was 13.8 months (95\% CI: 5.3-22.3; Figure 2C) and the median PFS was 8.7 months (95\% CI: 5.9-11.1; Figure 2D). There was no statistical difference in PFS for patients with PVTT (9.1 months) and without PVTT (4.1 months) $(\mathrm{P}=0.204)$. Patients without PVTT achieved a better prognosis than those with PVTT, with more than half of them still alive upon completion of the observation period. By comparison, the median OS for patients with PVTT was 5.1 months $(\mathrm{P}=0.007$; Figure $2 E)$. Therapeutic efficacy was significantly improved in patients with distant metastasis (DCR for patients with distant metastasis versus patients without distant metastasis, $81.3 \%$ versus $33.3 \%, \mathrm{P}=0.032$; PFS for patients with distant metastasis versus patients without distant metastasis, 9.9 versus 1.9 months, $\mathrm{P}=0.026$; Figure $2 F$ ). However, the median OS was not statistically significant between the two groups, with median OS for patients with distant metastasis at 13.8 months versus patients without distant metastasis at 6.0 months $(\mathrm{P}=0.237$; figure not shown).

\section{Safety}

The median time of apatinib treatment was 4.2 months, with 12 patients $(52.2 \%)$ completing $>4$ months of apatinib treatment, and only 2 of these 12 patients (16.7\%) experiencing disease progression. Eleven patients (47.9\%) took apatinib for $<4$ months, with 5 of these 11 patients (45.4\%) experiencing disease progression. Twentyone patients $(91.3 \%)$ required a dose reduction due to intolerance to AEs. Most AEs were mild and clinically acceptable (Table 3). The most common AEs were proteinuria (39.1\%), hypertension (34.8\%), hand-footskin reaction $(34.8 \%)$, and fatigue $(26.1 \%)$. Proteinuria and hypertension were also the most common grade $3 / 4$ AEs.

\section{Discussion}

Sorafenib and lenvatinib are the recommended first-line treatment for advanced HCC, according to the Barcelona Clinic Liver Cancer (BCLC) guidelines (3). However, the extended survival of patients treated with sorafenib in the SHARP and ORIENTAL clinical trials was limited to 2.8 and 2.3 months, respectively $(4,5)$. The efficacy of lenvatinib was not being inferior to sorafenib (6). Hence, a more effective treatment is critical for the treatment of 
A

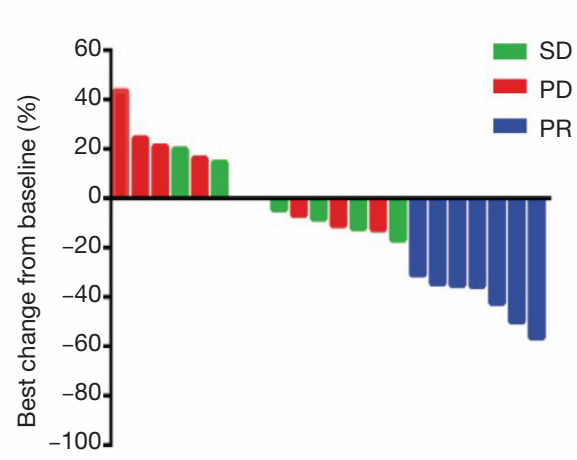

C

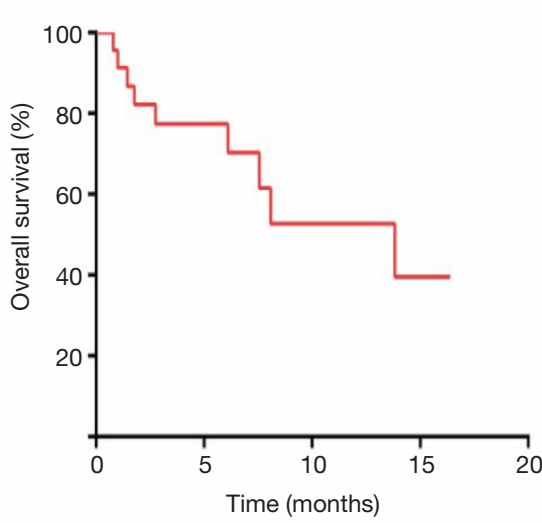

$E$

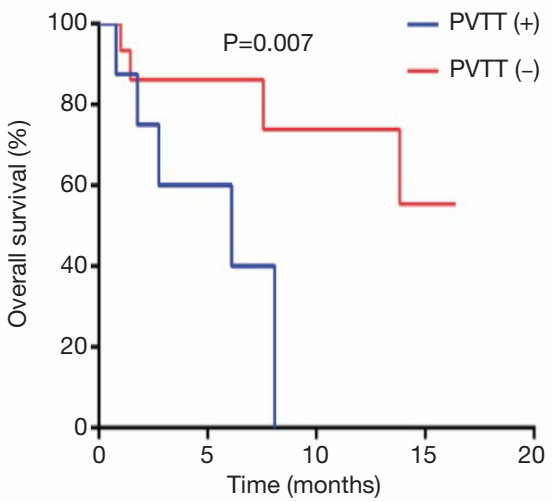

B

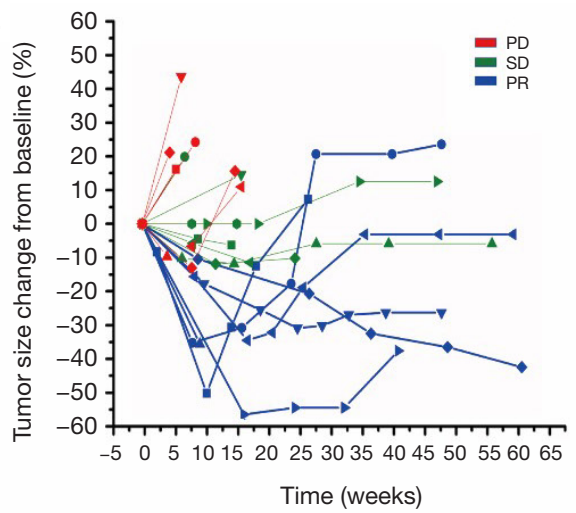

D
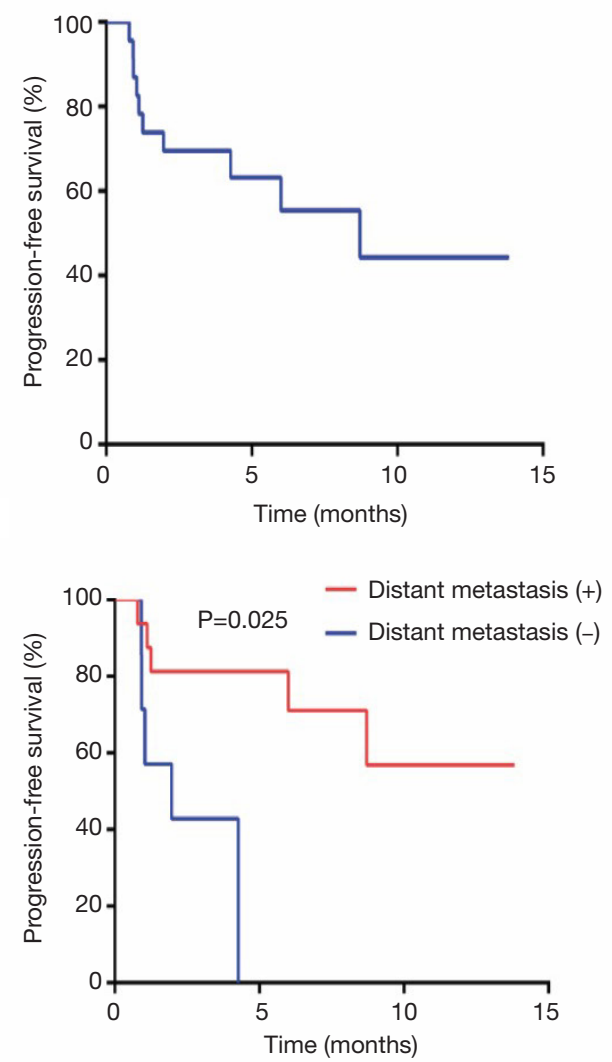

Figure 2 Changes in tumor size and the prognosis of all patients. (A) The waterfall plot for the best percentage change in target lesion size is shown for 22 patients who had at least one post-baseline efficacy assessment. (B) Changes in tumor size from baseline of patients who had at least one post-baseline efficacy assessment. (C,D) The median OS was 13.8 months (C). The median PFS was 8.7 months (D). (E) The median OS for patients with PVTT was 5.1 months; however, the median OS was not available as more than half of the patients without PVTT were still alive $(\mathrm{P}=0.007)$. (F) The median PFS was 1.9 months in patients without distant metastasis and 9.9 months in patients with distant metastasis. SD, stable disease; PD, progressive disease; PR, partial response; PVTT, portal vein tumor thrombosis; PFS, progressionfree survival; OS, overall survival. 
advanced HCC. Apatinib, a VEGFR2 inhibitor, was found to provide a novel therapeutic effect on advanced HCC in our retrospective study (17). The findings from the present phase II clinical trial further confirm the efficacy and safety of apatinib.

The SHARP and ORIENTAL clinical trials reported PR rates of only $2 \%$ and $3.3 \%$, respectively. In our study, however, PR and ORR were achieved in 7 patients (30.4\%). Recently, the REFLECT study reported an ORR for lenvatinib and sorafenib of $24 \%$ and $9.2 \%$, respectively (6). Therefore, this single-arm, open-label, single-center phase II clinical trial demonstrated that apatinib offers a significantly improved ORR compared to previously reported therapies and has acceptable toxicity in patients with advanced HCC.

Previous reports demonstrating the efficacy of apatinib

Table 2 Analysis of therapeutic efficacy

\begin{tabular}{lc}
\hline Characteristic & $\mathrm{N}(\%)$ \\
\hline $\mathrm{CR}$ & 0 \\
$\mathrm{PR}$ & $7(30.4)$ \\
$\mathrm{SD}$ & $8(34.8)$ \\
$\mathrm{PD}$ & $7(30.4)$ \\
$\mathrm{NE}$ & $1(4.3)$ \\
ORR (\%) & 30.40 \\
DCR (\%) & 65.20 \\
\hline
\end{tabular}

$\mathrm{CR}$, complete response; PR, partial response; SD, stable disease; PD, progressive disease; NE, no evaluated; ORR, objective response rate; $\mathrm{DCR}$, disease control rate. in treating HCC $(17,19-21)$ did not preclude selection bias, due to the retrospective nature of the studies. Furthermore, these reports incorporated efficacy rates of patients with combined antitumor therapies. This clinical trial was therefore designed to illustrate the efficacy of apatinib by its administration as a monotherapy to patients with advanced HCC, prior to disease progression. Interestingly, only three patients in this study received sorafenib treatment after disease progression. The finding of a sufficiently high PR rate and high DCR is noteworthy. For patients with advanced HCC, a high PR rate can provide an opportunity to receive alternative treatments, such as surgery or transarterial chemoembolization (TACE). This was observed in a previous clinical study where five patients with locally advanced HCC were initially unsuitable for resection. Administration of apatinib led to a significant $\mathrm{PR}$, reinstating their eligibility for radical resection. All five patients underwent this surgical procedure, with one patient ultimately achieving pathological CR. The data indicates that apatinib might be a suitable option for the treatment of patients with advanced HCC as first-line treatment, providing more opportunities for these patients to receive alternative treatments. This is especially applicable those with locally advanced HCC.

The median OS and PFS in this study were 13.8 and 8.7 months, respectively, which are comparable to the median OS and PFS reported for lenvatinib in the REFLECT study. However, it is worth noting that participants in our study with PVTT were in a more advanced stage of $\mathrm{HCC}(3 \mathrm{Vp} 4$ and $5 \mathrm{Vp} 3$ ). The median OS for patients with PVTT was only 5.1 months. By contrast, the median OS for patients without PVTT was not

Table 3 Analysis of adverse events

\begin{tabular}{lcc}
\hline Adverse event & All grades, $\mathrm{n}(\%)$ & Grade 3/4, $\mathrm{n}(\%)$ \\
\hline Hypertension & $8(34.8)$ & $3(13.0)$ \\
Proteinuria & $9(39.1)$ & $3(13.0)$ \\
Hand-foot-skin reaction & $8(34.8)$ & $1(4.3)$ \\
Fatigue & $6(26.1)$ & 0 \\
Diarrhea & $4(17.4)$ & $1(4.3)$ \\
Pain & $4(17.4)$ & 0 \\
Nausea and vomiting & $5(21.7)$ & 0 \\
Increased transaminase & $6(26.1)$ & 0 \\
Anorexia & $5(21.7)$ & 0 \\
\hline
\end{tabular}


available as more than half of these patients were still alive at the end of the study (Figure 2E). The PFS for patients without distant metastasis was only 1.9 months (Figure $2 F$ ). By contrast, in the REFLECT study, patients with a more advanced PVTT stage were excluded. In addition, 3 patients in that study received sorafenib treatment after disease progression, and 20 received only supportive treatment, thereby affecting the median OS. Therefore, we believe that treatment with apatinib could have provided a better prognosis if patients with more advanced PVTT were excluded. Notably, 5 of 8 (62.5\%) patients with PVTT, 8 of $12(66.7 \%)$ patients with lymph node metastasis, and 8 of 9 (88.9\%) patients with distant metastasis achieved PR or SD. This suggests that apatinib was highly effective in the treatment of patients with advanced HCC, especially those with metastases (Figure $2 F$ ).

In our study, proteinuria (39.1\%), hypertension (34.8\%), hand-foot-skin reaction (34.8\%), and fatigue (26.1\%) were the most common AEs. The most common grade 3/4 AEs were proteinuria and hypertension. Proteinuria and hypertension are the most common AEs reported for patients treated with antiangiogenic drugs (22-24). However, the specific mechanisms underlying the development of hypertension and proteinuria with apatinib treatment are unknown. Some studies have suggested that the occurrence of these AEs is dose-dependent (25-27). In this study, we also found that the occurrence of AEs was related to a higher dose of apatinib. These AEs decreased and became easier to manage when the apatinib dose was adjusted to $250 \mathrm{mg}$. Thus, apatinib generally has an acceptable safety profile as an antiangiogenic agent.

The recommended oral dose of apatinib for advanced gastric adenocarcinoma or gastroesophageal junction adenocarcinoma is $850 \mathrm{mg} /$ day (13). However, as most patients with HCC have liver cirrhosis and hepatic insufficiency, we set the initial dose in our study to $500 \mathrm{mg}$, once daily. Most patients (21/23) could not tolerate serious apatinib-related AEs. However, only one participant in this study required a dose interruption in patients receiving a low dose $(250 \mathrm{mg})$ of apatinib. More importantly, the therapeutic effect of apatinib treatment was clinically acceptable, despite the low dose. The mechanism of apatinib may not be limited to the anti-VEGFR2 effect. One study showed that a low dose of apatinib can reshape the immunosuppressed tumor microenvironment into a permissive anti-tumor immunity (28) and may also contribute to a high response rate. Therefore, we propose that an initial daily dose of $250 \mathrm{mg}$ may be appropriate for apatinib treatment in patients with advanced HCC.

Over the past few years, the focus on immunotherapy has broadened and intensified. Immunotherapy has likewise contributed to breakthroughs in the field of advanced HCC. Among these is PD-1 monoclonal antibody which has been approved by the Food and Drug Administration for treating advanced HCC (29). Recent studies have shown that the combination of PD-1 antibody and anti-angiogenesis therapy may be a better approach to treating HCC, achieving ORRs of 40-50\% (30,31). At the ESMO 2018 congress, it was reported that PD-L1 monoclonal antibody combined with bevacizumab could achieve an ORR of $34 \%$. Additionally, at the ASCO GI 2019 symposium, a 37.5\% pathological CR was reported when CTLA-4 antibody was combined with PD-1 monoclonal antibody for neoadjuvant therapy. These studies provide new hope for improving the curative effect of HCC, especially locally advanced or resectable HCC $(32,33)$. However, how to choose the combination of drugs has still not yet been determined. The therapeutic effect of immunotherapy may be enhanced by apatinib, as it can optimize the tumor microenvironment. Meanwhile, our study showed that apatinib has a satisfactory therapeutic effect, and is thus a strong contender for future combination therapies.

Although satisfactory results were obtained with apatinib treatment for advanced HCC in our study, several improvements can still be made. Firstly, a larger scale, randomized phase III clinical trial is required to confirm the findings presented in this article. Secondly, biomarkers are needed to identify patients who are most likely to benefit from apatinib. Furthermore, the mechanism of apatinib resistance is still unknown, and understanding how apatinib resistance can be overcome remains a major clinical challenge. At last, it also remains unclear whether apatinib combined with TACE or other modalities can improve the efficacy for treating advanced HCC (34).

\section{Conclusions}

In conclusion, the findings of our phase II clinical trial support the use of apatinib as a new treatment option for patients with advanced HCC, with moderate, reversible, and acceptable AEs.

\section{Acknowledgments}

This article has been edited by Professor James Liu. We are grateful to all the sponsors for their support. We also thank 
the independent data monitoring committee led by Nayun Wei.

Funding: This study was supported by the National Natural Science Foundation of China (81672884), the National Science and Technology Major Project (2017ZX10203207), and the Chinese Anti-Cancer Association-Heng Rui Pharmaceutical Innovation Research Fund.

\section{Footnote}

Reporting Checklist: The authors have completed the TREND reporting checklist. Available at http://dx.doi. org/10.21037/atm-20-2990

Data Sharing Statement: Available at http://dx.doi. org/10.21037/atm-20-2990

Conflicts of Interest: All authors have completed the ICMJE uniform disclosure form (available at http://dx.doi. org/10.21037/atm-20-2990). The authors have no conflicts of interest to declare.

Ethical Statement: The authors are accountable for all aspects of the work in ensuring that questions related to the accuracy or integrity of any part of the work are appropriately investigated and resolved. The study was conducted in accordance with the Declaration of Helsinki (as revised in 2013). The study was approved by the Ethics Committee of the Tianjin Medical University Cancer Institute and Hospital and is registered on clinicaltrials.gov (NCT03046979) and informed consent was taken from all the patients.

Open Access Statement: This is an Open Access article distributed in accordance with the Creative Commons Attribution-NonCommercial-NoDerivs 4.0 International License (CC BY-NC-ND 4.0), which permits the noncommercial replication and distribution of the article with the strict proviso that no changes or edits are made and the original work is properly cited (including links to both the formal publication through the relevant DOI and the license). See: https://creativecommons.org/licenses/by-nc-nd/4.0/.

\section{References}

1. Liu K, McCaughan GW. Epidemiology and etiologic associations of non-alcoholic fatty liver disease and associated HCC. Adv Exp Med Biol 2018;1061:3-18.
2. Zhu AX, Fuchs CS, Clark JW, et al. A phase II study of epirubicin and thalidomide in unresectable or metastatic hepatocellular carcinoma. Oncologist 2005;10:392-8.

3. European Association for the Study of the Liver. EASL Clinical Practice Guidelines: Management of hepatocellular carcinoma. J Hepatol 2018;69:182-236.

4. Llovet JM, Ricci S, Mazzaferro V, et al. Sorafenib in advanced hepatocellular carcinoma. N Engl J Med. 2008;359:378-90.

5. Cheng AL, Kang YK, Chen Z, et al. Efficacy and safety of sorafenib in patients in the Asia-Pacific region with advanced hepatocellular carcinoma: a phase III randomised, double-blind, placebo-controlled trial. Lancet Oncol 2009;10:25-34.

6. Kudo M, Finn RS, Qin S, et al. Lenvatinib versus sorafenib in first-line treatment of patients with unresectable hepatocellular carcinoma: a randomised phase 3 noninferiority trial. Lancet 2018;391:1163-73.

7. Spannuth WA, Sood AK, Coleman RL. Angiogenesis as a strategic target for ovarian cancer therapy. Nat Clin Pract Oncol 2008;5:194-204.

8. Cross MJ, Claesson-Welsh L. FGF and VEGF function in angiogenesis: signalling pathways, biological responses and therapeutic inhibition. Trends Pharmacol Sci 2001;22:201-7.

9. Shinkaruk S, Bayle M, Lain G, et al. Vascular endothelial cell growth factor (VEGF), an emerging target for cancer chemotherapy. Curr Med Chem Anticancer Agents 2003;3:95-117.

10. Veeravagu A, Hsu AR, Cai W, et al. Vascular endothelial growth factor and vascular endothelial growth factor receptor inhibitors as anti-angiogenic agents in cancer therapy. Recent Pat Anticancer Drug Discov. 2007;2:59-71.

11. Kim YM, Hwang S, Kim YM, et al. Endostatin blocks vascular endothelial growth factor-mediated signaling via direct interaction with KDR/Flk-1. J Biol Chem 2002;277:27872-9.

12. Price DJ, Miralem T, Jiang S, et al. Role of vascular endothelial growth factor in the stimulation of cellular invasion and signaling of breast cancer cells. Cell Growth Differ 2001;12:129-35.

13. Li J, Qin S, Xu J, et al. Randomized, Double-Blind, Placebo-Controlled Phase III Trial of Apatinib in Patients With Chemotherapy-Refractory Advanced or Metastatic Adenocarcinoma of the Stomach or Gastroesophageal Junction. J Clin Oncol 2016;34:1448-54.

14. Lan CY, Wang Y, Xiong Y, et al. Apatinib combined with oral etoposide in patients with platinum-resistant 
or platinum-refractory ovarian cancer (AEROC): a phase 2, single-arm, prospective study. Lancet Oncol 2018;19:1239-46.

15. Wu F, Zhang S, Gao G, et al. Successful treatment using apatinib with or without docetaxel in heavily pretreated advanced non-squamous non-small cell lung cancer: A case report and literature review. Cancer Biol Ther 2018;19:141-4.

16. Hu X, Zhang J, Xu B, et al. Multicenter phase II study of apatinib, a novel VEGFR inhibitor in heavily pretreated patients with metastatic triple-negative breast cancer. Int J Cancer 2014;135:1961-9.

17. Kong Y, Sun L, Hou Z, et al. Apatinib is effective for treatment of advanced hepatocellular carcinoma. Oncotarget 2017;8:105596-605.

18. Liver Cancer Study Group of Japan. The general rules for the clinical and pathological study of primary liver cancer. Liver Cancer Study Group of Japan. Jpn J Surg 1989;19:98-129.

19. Fan W, Yuan G, Fan H, et al. Apatinib Combined With Transarterial Chemoembolization in Patients With Hepatocellular Carcinoma and Portal Vein Tumor Thrombus: A Multicenter Retrospective Study. Clin Ther 2019;41:1463-76.

20. Yang Z, Chen G, Cui Y, et al. The safety and efficacy of TACE combined with apatinib on patients with advanced hepatocellular carcinoma: a retrospective study. Cancer Biol Ther 2019;20:321-7.

21. Zhu Y, Feng B, Mei L, et al. Clinical efficacy of TACE combined with Apatinib in the treatment of advanced hepatocellular carcinoma. J BUON 2019;24:608-14.

22. Kappers $M H$, van Esch JH, Sleijfer S, et al. Cardiovascular and renal toxicity during angiogenesis inhibition: clinical and mechanistic aspects. J Hypertens 2009;27:2297-309.

23. van Heeckeren WJ, Ortiz J, Cooney MM, et al. Hypertension, proteinuria, and antagonism of vascular endothelial growth factor signaling: clinical toxicity, therapeutic target, or novel biomarker? J Clin Oncol 2007;25:2993-5.

Cite this article as: Hou Z, Zhu K, Yang X, Chen P, Zhang W, Cui Y, Zhu X, Song T, Li Q, Li H, Zhang T. Apatinib as first-line treatment in patients with advanced hepatocellular carcinoma: a phase II clinical trial. Ann Transl Med 2020;8(17):1047. doi: 10.21037/atm-20-2990
24. Izzedine H, Massard C, Spano JP, et al. VEGF signalling inhibition-induced proteinuria: Mechanisms, significance and management. Eur J Cancer 2010;46:439-48.

25. Eskens FA, Verweij J. The clinical toxicity profile of vascular endothelial growth factor (VEGF) and vascular endothelial growth factor receptor (VEGFR) targeting angiogenesis inhibitors; a review. Eur J Cancer 2006;42:3127-39.

26. Launay-Vacher V, Deray G. Hypertension and proteinuria: a class-effect of antiangiogenic therapies. Anticancer Drugs 2009;20:81-2.

27. Féliz LR, Tsimberidou AM. Anti-vascular endothelial growth factor therapy in the era of personalized medicine. Cancer Chemother Pharmacol 2013;72:1-12.

28. Zhao S, Ren S, Jiang T, et al. Low-dose apatinib optimizes tumor microenvironment and potentiates antitumor effect of PD-1/PD-L1 blockade in lung cancer. Cancer Immunol Res 2019;7:630-43.

29. Villanueva A. Hepatocellular Carcinoma. N Engl J Med 2019;380:1450-62.

30. Makker V, Rasco D, Vogelzang NJ, et al. Lenvatinib plus pembrolizumab in patients with advanced endometrial cancer: an interim analysis of a multicentre, open-label, single-arm, phase 2 trial. Lancet Oncol 2019;20:711-8.

31. Xu J, Zhang Y, Jia R, et al. Anti-PD-1 antibody SHR1210 combined with apatinib for advanced hepatocellular carcinoma, gastric, or esophagogastric junction cancer: An open-label, dose escalation and expansion study. Clin Cancer Res 2019;25:515-23.

32. Kudo M. Systemic Therapy for Hepatocellular Carcinoma: Latest Advances. Cancers (Basel) 2018;10:412-28.

33. El Dika I, Khalil DN, Abou-Alfa GK. Immune checkpoint inhibitors for hepatocellular carcinoma. Cancer 2019;125:3312-9.

34. Chen S, Yu W, Zhang K, et al. Comparison of the efficacy and safety of Transarterial chemoembolization with and without Apatinib for the treatment of BCLC stage C hepatocellular carcinoma. BMC Cancer 2018;18:1131-2. 